\title{
Heat shock response and insulin-associated neurodegeneration
}

\author{
Michael J. Urban ${ }^{1}$, Rick T. Dobrowsky ${ }^{2}$, and Brian S.J. Blagg ${ }^{3}$ \\ ${ }^{1}$ Neuroscience Graduate Program, The University of Kansas, Lawrence, KS 66045, USA \\ 2 Department of Pharmacology and Toxicology, The University of Kansas, Lawrence, KS 66045, \\ USA
}

${ }^{3}$ Department of Medicinal Chemistry, The University of Kansas, Lawrence, KS 66045, USA

\begin{abstract}
Dysfunctional insulin and insulin-like growth factor-I (IGF-I) signaling contributes to the pathological progression of diabetes, diabetic peripheral neuropathy (DPN), Alzheimer's (AD), Parkinson's (PD) and Huntington's diseases (HD). Despite their prevalence, there are limited therapeutic options available for the treatment of these neurodegenerative disorders. Therefore, establishing a link between insulin/IGF-I and the pathoetiology of these diseases may provide alternative approaches toward their management. Many of the heat shock proteins (Hsps) are wellknown molecular chaperones that solubilize and clear damaged proteins and protein aggregates. Recent studies suggest that modulating Hsps may represent a promising therapeutic avenue for improving insulin and IGF-I signaling. Pharmacological induction of the heat shock response (HSR) may intersect with insulin/IGF-I signaling to improve aspects of neurodegenerative phenotypes. Herein, we review the intersection between Hsps and the insulin/IGF systems under normal and pathological conditions. The discussion will emphasize the potential of non-toxic HSR inducers as viable therapeutic agents.
\end{abstract}

\section{Diabetic neuropathy and heat shock proteins}

The administration of extracted pancreatic insulin in 1922 marked the first effective therapeutic option to combat diabetes and associated peripheral neuropathy. Although controlled insulin therapy significantly decelerates the rate of progression of acute and chronic diabetic complications, they still develop and represent a significant risk to the overall deterioration of the health of the individual. DPN is the overall attrition of sensory nerve fibers that results from diabetes. Treatments for DPN generally offer monosymptomatic relief (e.g. analgesics to mitigate painful neuropathy) or specifically inhibit single, putative pathogenic mechanisms associated with diabetes-induced hyperglycemia [e.g. antioxidants, aldose reductase inhibitors, and poly(ADP-ribose) polymerase (PARP) inhibitors] [1,2]. Mismanaged insulin therapy can also generate symptoms of 'insulin shock' (acute hyperinsulinism), depriving the brain of essential glucose, or diabetic encephalopathy (hypoinsulinism) [3]. For this reason, treatment of DPN can benefit from a more multifaceted therapeutic approach. In addition to managing non-

(C) 2011 Elsevier Ltd. All rights reserved.

Corresponding author: Blagg, B.S.J. (bblagg@ku.edu)..

Publisher's Disclaimer: This article appeared in a journal published by Elsevier. The attached copy is furnished to the author for internal non-commercial research and education use, including for instruction at the authors institution and sharing with colleagues. Other uses, including reproduction and distribution, or selling or licensing copies, or posting to personal, institutional or third party websites are prohibited. In most cases authors are permitted to post their version of the article (e.g. in Word or Tex form) to their personal website or institutional repository. Authors requiring further information regarding Elsevier's archiving and manuscript policies are encouraged to visit: http://www.elsevier.com/copyright 
neuronal postprandial glucose uptake, insulin and IGF signaling are required during early neurological development and to maintain neuronal viability and function throughout the nervous system [4-7]. Several components of insulin and insulin-like growth factor-I (IGFI) signaling, including insulin receptors (commonly mutated in insulin-resistant patients) and IGF-I receptors, rely upon heat shock proteins (Hsps) for post-translational modifications and integrity [8-11]. Here we review Hsps and their role within normal and pathological insulin/IGF systems with an emphasis upon their potential as therapeutic targets.

\section{The heat shock response}

Several Hsps serve as molecular chaperones that assist in the folding of nascent polypeptides, or 'client proteins', into their mature conformations [12-15]. These chaperones also assist in the refolding of damaged proteins that arise under stressed conditions, such as nutrient deprivation, oxidative and nitrosative stress, and assorted insults to the cell [12-15]. Additionally, Hsps serve as intracellular triage units that refold damaged proteins, tag irreparable proteins for proteolytic degradation, stabilize protein complexes, solubilize protein aggregates and facilitate intracellular trafficking [12-16].

\section{Heat shock protein 90 (Hsp90) and heat shock factor 1 (HSF1)}

Hsp90 is a $90 \mathrm{kDa}$ homodimeric cytosolic protein that interacts with more than 200 client proteins and $\sim 50$ co-chaperones [12-15,17]. Although all the molecular details by which Hsp90 folds client proteins remain unresolved, the molecular components of the folding machinery have been well-studied. The co-chaperones Hsp70 and Hsp40 bind polypeptide substrates and are recruited into a heteroprotein complex with Hsp90 and Hsp70/Hsp90organizing protein (HOP) [17]. This stabilized complex enables client protein transfer to the Hsp90 homodimer, thereby permitting Hsp70, Hsp40 and HOP dissociation in some cases [17]. Additional co-chaperones and immunophilins aid ATP binding within the Hsp90 Nterminal nucleotide-binding domain, promoting $\mathrm{N}$-termini dimerization and 'clamping' of the client protein [17]. Co-chaperone p23 further stabilizes the clamped protein complex and modulates nucleotide hydrolysis by altering the intrinsic ATPase activity of Hsp90 [17]. ATPase homolog 1 (AHA1) then initiates ATP hydrolysis to produce the energy necessary to induce conformational changes essential to client protein folding and release [17]. $\mathrm{N}$ terminal Hsp90 inhibitors function by blocking ATP-mediated dimerization and clamping of the client protein, destabilizing the complex and prematurely releasing the client protein [18]. Failure to produce mature client proteins is typically cytotoxic because cellular levels can decrease following ubiquitination, and proteasomal degradation of improperly folded proteins $[17,18]$.

Chaperones are also essential for the refolding of aggregated, damaged and denatured proteins. Under normal conditions, Hsp90 binds HSF1, and this complex prevents the HSR $[19,20]$. Upon stress or heat shock, Hsp90 releases HSF1 and ultimately induces the HSR following phosphorylation, trimerization and translocation into the nucleus [19,20]. The HSR is characterized, in part, by the increased expression of Hsp27, Hsp40, Hsp70, Hsp90 and other genes $[19,20]$. Pharmacologically, small-molecule Hsp90 inhibitors mimic cellular stress by disrupting the Hsp90:HSF1 complex [21].

Therapeutically, the development of N-terminal Hsp90 inhibitors may provide effective chemotherapeutic opportunities because numerous Hsp90 client proteins are also oncoproteins (e.g. Akt, ErbB2, Bcr-Abl, mutant p53 and v-Src) [12,13,22]. As such, inhibiting Hsp90 is an attractive target for cancer therapy because it simultaneously targets multiple molecular components associated with all six hallmarks of cancer [12,23]. However, demonstrating chemotherapeutic efficacy has been confounded by the narrow therapeutic window associated with these inhibitors [13]. N-terminal inhibitors, such as the 
benzoquinone ansamycin antibiotics geldanamycin (GDA) and the structural analog 17(allylamino)-17-demethoxy geldanamycin (17-AAG) (Figure 1), fail to generate therapeutic windows that dissociate client protein degradation from HSR induction [13]. Because HSR induction leads to Hsp70 and Hsp90 overexpression, these pro-survival Hsps antagonize the desired endpoint of cytotoxicity in cancer cells by increasing the folding/refolding of the targeted oncoproteins [12-15,23]. Furthermore, both of these compounds exhibit hepatotoxicity in preclinical (GDA) or clinical $(17-\mathrm{AAG})$ trials $[13,15]$. Other N-terminal inhibitors, such as celastrol, gedunin and $\mathrm{H}_{2}$-gamendazole (Figure 1), also induce client protein degradation by disrupting the interface between Hsp90 and the kinase co-chaperone Cdc37 (cell division cycle 37 homolog, Saccharomyces cerevisiae), but still suffer from an overlap in concentrations that promote cytoprotective and cytotoxic responses [24].

In contrast to selecting for cytoxicity, it has been proposed that HSR induction can promote the disaggregation of misfolded proteins associated with several neurodegenerative diseases. In this regard, $\mathrm{N}$-terminal inhibitors decrease tau and $\mathrm{Ab}$ aggregation in $\mathrm{AD}$, improve motor function in spinal and bulbar muscular atrophy and prevent mutant huntingtin (mHtt) aggregation in HD [25-29]. However, similar to cancer therapies, selecting for neuroprotection still entails having a sufficient therapeutic window to minimize potential cytotoxicity. This can be accomplished through C-terminal Hsp90 inhibition.

The C-terminus of Hsp90 contains an additional nucleotide-binding domain that also binds the natural product novobiocin (Figure 1), a DNA gyrase ATP binding-site inhibitor [30]. Although novobiocin itself is a poor inducer of client protein degradation and the HSR, higher affinity and more potent C-terminal Hsp90 inhibitors have been developed around the coumarin scaffold of novobiocin [30,31]. Through structure-activity relationship studies, induction of the HSR and associated cytoprotection have been divested from client protein degradation and cytotoxicity, thus expanding the dose range for neuroprotection.

Representative examples are A4 and KU-32 [N-(7-((2R,3R,4S,5R)-3,4-dihydroxy-5methoxy-6,6-dimethyl-tetrahydro-2H-pyran-2-yloxy)-2-oxo-2-methyl-chromen-3yl)acetamide] (Figure 1), which protects against A $\beta$-induced death of cortical neurons, glucose-induced cell death of unmyelinated sensory neurons and agonist-induced demyelination of myelinated sensory neurons [32-34]. Moreover, KU-32 has proved to be effective at reversing sensory hypoalgesia and nerve conduction deficits associated with DPN in streptozotocin (STZ)-induced type 1 diabetic mice without any overt cytotoxicity [34].

Chemical induction of the HSR can also be achieved independently of Hsp90 inhibition. For example, HSF1A (Figure 1) induces the HSR through apparent interactions with the chaperonin TRiC [TCP1 (tailless complex polypeptide 1)-ring complex] independently of Hsp90 binding [35]. In NG108-15 neuroprogenitor cells, riluzole (used to treat amyotrophic lateral sclerosis, ALS) administration slows HSF1 turnover while amplifying HSF1 and glutamate transporter (GLT1) expression/activation [36]. GLT1 enhances glutamate recovery from the synaptic cleft, reducing the likelihood of glutamate excitotoxicity (implicated in painful diabetic neuropathy, ALS, AD, PD and HD) [36]. However, the specific mechanisms underlying HSR induction by HSF1A and riluzole remain undetermined $[35,36]$. By contrast, compounds such as quercetin can disrupt the DNA:HSF1 binding interface, preventing the transcription of Hsp mRNA (especially Hsp70) [37].

HSF1 also regulates several genes in the absence of heat shock. For example, downregulating HSF1 in HeLa cervical carcinoma cells with small interfering RNA (siRNA) modulated the expression of 378 genes involved in cell cycle regulation and cell proliferation [38]. This suggests that HSF1 maintains the expression of several proteins required for survival under stressful (i.e. cancerous) conditions in addition to heat-shock- 
inducible Hsps [20,38]. Under non-oncogenic conditions, HSF1 cooperates with NFATC2 (nuclear factor of activated T-cells-2) to regulate the expression of $\alpha \mathrm{B}$-crystallin (small heat shock protein) and the scaffolding protein PDZK3 [PSD-95/Dlg-A/ZO-1 (PDZ) domaincontaining-3] [39]. This enables $\alpha \mathrm{B}$-crystallin-mediated disaggregation of mutant huntingtin in R6/2 HD mice [39]. In the insulin-like signaling pathway of Caenorhabditis elegans, the FoxO (forkhead box 'other') family transcription factor DAF-16 (abnormal dauer formation-16) collaborates with HSF1 to induce the expression of genes that increase life expectancy [19]. Hence, HSF1 activation enhances cell survival under both normal and stressed conditions.

\section{Hsp70, Hsp40 and Hsp27}

Independently of Hsp90, Hsp70 and Hsp40 form heteroprotein complexes that offer additional neuroprotective attributes. J-domain-containing proteins (J proteins), such as Hsp40 and CSP $\alpha$ (cysteine string protein $\alpha$ ), bind constitutive Hsc70 (heat shock cognate 70) and inducible Hsp70 (Hsp72) to enhance the ATPase activity of Hsp70 [40]. Although binding affinities differ among Hsp70 isoforms, there is a degree of interchangeability with assorted J proteins [40]. Specialized neuronal J protein:Hsp70 interactions repair damaged synaptic vesicle components, enable vesicular secretion/reuptake (e.g. calcium channel regulation, clathrin exchange), permit guanine nucleotide exchange factor activities (e.g. $\mathrm{CSPa}$ ) associated with G-protein-coupled receptors, solubilize potentially toxic aggregates and assist in protein ubiquitination [40].

Hsp70 and Hsc70 interact with BAG-1 (Bcl-2-associated athanogene) and CHIP (Cterminus of $\mathrm{Hsc} 70 / \mathrm{Hsp} 70$-interacting protein) to flag irreparable proteins with ubiquitin for proteasomal degradation [41]. Alternatively, BAG-2 enables ubiquitin-independent Hsp70mediated delivery of irreparable substrates directly to $20 \mathrm{~S}$ proteasomes for degradation [42]. Upon Hsp70/BAG-2 saturation, clearance duties are reinforced by the Hsp70/BAG-1/CHIP complex [42]. Such triage management has been implicated in tau clearance (AD), where effective Hsp70 chaperoning becomes exhausted [42]. These data support that targeting select Hsp70 isoforms via chemical modulators may enhance desired neuronal J protein interactions and disrupt unfavorable associations (e.g. tau triage), collectively improving neuronal function [37,43]. Although selective inhibition of Hsp70 isoforms provides promise as neuroprotective and anti-cancer therapeutics, characterization of isoform selective scaffolds that modulate Hsp70 (e.g. dihydropyrimidines, polyamines and ATP mimics) are still underway [37,43].

\section{Insulin and IGF systems}

To assess the importance of Hsps within the insulin/IGF signaling cascades, ligand bioavailability, receptor localization and signaling components are reviewed below. Insulin bioavailability within the periphery depends upon secretion from pancreatic $\beta$ cells in the islets of Langerhans. Brain insulin levels rely upon endothelial facilitated transport across the blood-brain barrier (BBB) into the cerebrospinal fluid (CSF). These levels are regulated independently of plasma insulin fluctuations (e.g. postprandial insulin secretion) [44]. Under acute hyperinsulinemia, insulin translocation is limited by endothelial saturation, whereas chronic hyperinsulinemia (e.g. insulin resistance) results in receptor internalization [45]. Mammalian brain neurons can also endogenously synthesize small amounts of insulin to sustain homeostatic activities [46].

IGF-I and IGF-II are abundantly expressed in developing brains. In adults, endogenous IGFI is diminished and the brain relies primarily on circulating IGF-I [47]. Upon crossing the BBB, IGF-I can modulate neuronal growth and angiogenesis [47]. IGF-II is more highly expressed in adult brain (especially the hippocampus), but its production slowly declines 
with age [48]. In the periphery, IGF-I and IGF-II signaling supports both motor and sensory nerve viability and governs neuronal migration and neuritogenesis, enabling target tissue innervation during development and nerve regeneration as an adult [7]. IGF-I is localized to the ventral horn, dorsal root and sympathetic ganglia, Schwann cells and target tissues (e.g. skeletal muscle) [7]. Schwann cells and skeletal muscle also provide IGF-II for neurotrophic support [7].

\section{Insulin receptor (IR) and IGF-I receptor (IGF-IR) expression}

The distribution patterns of the IR and IGF-IR are distinct within the mammalian nervous system and correlate with several areas implicated in AD, PD, HD and DPN (Table 1) [7,49,50]. Structurally, IR and IGF-IR are tetrameric proteins consisting of two extracellular ligand-binding domains ( $\alpha$ subunits) and two receptor tyrosine kinase (RTK) domains ( $\beta$ subunits) [5]. Interestingly, molecular differences between receptor compositions can affect ligand binding affinities, and in adipose, muscle and liver the metabolic effects of insulin are enhanced through augmented expression of the IR-B isoform over the IR-A isoform [5]. Disruption of relative IR-B:IR-A expression levels in these tissues is hypothesized to contribute to insulin resistance associated with type 2 diabetes [5]. IR-A is the main insulin receptor isoform in nerves and exhibits higher binding affinity for insulin, IGF-I and IGF-II compared to IR-B (Table 2 for $\mathrm{IC}_{50}$ values) [5,51,52]. IGF-I/II binding to IR-A (and IGFIR) selectively induces mitogenic effects via the MAPK/ERK (mitogen-activated protein kinase/extracellular-signal-regulated kinase) signaling pathway, suggesting an element for differential activation [5]. However, the effects elicited through insulin binding to IR-A are not typically metabolic, as seen with IR-B, but enhance viability and regulate biological functions such as synaptic transmission and plasticity [5].

\section{IR and IGF-IR signaling}

Ligand-induced IR/IGF-IR autophosphorylation enables its RTK domain to phosphorylate key regulatory substrates [e.g. IRS-1 (insulin receptor substrate-1), IRS-2 or Shc (Src homology 2 domain-containing)] that activate the MAPK/ERK, mTOR/raptor (mammalian target of rapamycin/regulatory-associated protein of mTOR) and PI3K (phosphatidylinositol 3-kinase) pathways (Figure 2) [5]. Induction of the MAPK/ERK pathway via receptoractivated Ras or Rheb (brain isoform) favorably enhances transcription factors that promote cell growth, proliferation and survival (e.g. c-Fos and c-Myc) [5]. Ras/Rheb also enhances mTOR/raptor signaling, stimulating p70S6 kinase- and eIF4E (eukaryotic translation initiation factor 4E)-regulated cell growth, autophagy, metabolism, ribosome biogenesis and translation [5]. PI3K pathway induction synergistically enhances MAPK/ERK and mTOR/ raptor signaling via Akt (protein kinase B) phosphorylation [5]. Activated Akt phosphorylates TSC2 (tuberous sclerosis complex 2) and prevents GTP hydrolysis by bound Ras/Rheb [5]. Furthermore, Akt-mediated MDM2 (murine double minute-2) phosphorylation enables p53 sequestration and ubiquitination, consequently reducing the expression of TSC2, IGF-binding protein-3 (prevents IGF binding to IGF-IR) and PTEN [phosphatase and tensin homolog; dephosphorylates PIP $_{3}$ (phosphatidylinositol 3,4,5triphosphate)] [53].

The importance of Akt in cell function and viability is far-reaching and impacts nonneuronal and neuronal function and viability. For example, within the periphery, Akt enhances GLUT4 (glucose transporter 4) vesicle transit velocity and membrane docking/ integration required for glucose transport into IR-B-expressing tissues, thereby increasing postprandial glucose clearance [54]. Interestingly, GLUT4 also localizes within several IRexpressing regions of the brain (i.e. cerebellum, sensorimotor cortex, hippocampus, pituitary and hypothalamus), suggesting a metabolic role for insulin [49]. Akt also mitigates proapoptotic signaling by flagging the FoxO3a (forkhead box 'other' 3 a) transcription factor 
for ubiquitination, reducing Fas ligand stimulation of the extracellular death-receptor pathway [5]. Akt-driven BAD phosphorylation disrupts BAD tetramerization, preventing mitochondrial permeabilization and initiation of the mitochondrial death pathway [5].

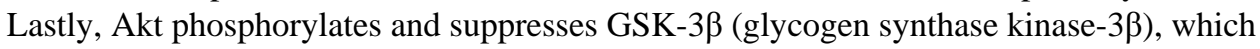
further enhances mitochondrial permeabilization through BAX phosphorylation [55].

\section{Signaling effects upon synaptic transmission and plasticity}

Although insulin/IGF-I signaling offers generalized neuroprotection, this signaling can also modulate neuron-specific synaptic plasticity. For example, insulin/IGF-I signaling significantly modulates glutamate ( $N$-methyl-D-aspartic acid, NMDA)-induced excitatory signaling within the peripheral and central nervous systems. In painful neuropathy studies, STZ-induced diabetic rats overexpress and activate metabotropic glutamate receptors (mGluR5), thereby amplifying afferent nociceptive signaling from sensory dorsal root ganglia (DRG) [56]. Lumbar spinal sections from STZ-induced hyperalgesic diabetic rats also exhibit amplified phosphorylation and activation of the NR1 subunit of the NMDA receptor (NMDAR) [57]. In this regard it is currently hypothesized that NMDAR activation induces the MAPK/ERK pathway and provides an ERK-dependent positive feedback loop that leads to NR1 phosphorylation [57]. However, the specific molecular components associated with this phosphorylation remain unclear. Furthermore, $\mathrm{GABA}_{\mathrm{B}}(\gamma$-aminobutyric acid, class B) receptor expression is significantly reduced in the lumbar spinal cords of STZinduced diabetic rats, suggesting that GABAergic inhibitory signaling required for the suppression of nociceptive transmission may be compromised [58]. Collectively, these data suggest that insulin deprivation in the development of diabetes can induce glutamatergic sensitization, thereby contributing to neuropathic pain. In hippocampal neurons, long-term potentiation (LTP) of NMDAR activity is hypothesized to facilitate memory formation [59]. Insulin/IGF-I signaling enhances NMDAR integration into the plasma membrane and induces the phosphorylation/activation of NR2A and NR2B subunits of NMDARs via protein kinase $\mathrm{C}$ (PKC) activation [59,60]. NR2A and NR2B expression is down-regulated in $\mathrm{AD}$, decreasing the likelihood of NMDAR potentiation [59]. Further, oligomeric $\mathrm{A} \beta$ preferentially activates NR2A over NR2B, generating an aberrantly high NR2A:NR2B ratio that favors long-term depression (LTD), potentially antagonizing memory establishment $[59,61]$.

Another insulin-induced alteration to synaptic plasticity is its effect upon the reuptake of norepinephrine, an essential neurotransmitter within the sympathetic nervous system that also impacts upon attention, mood and memory in the brain. Norepinephrine reuptake is facilitated via presynaptic norepinephrine transporters. These transporters are significantly reduced in the lumbar spinal cords of both type 1 and type 2 diabetic rats as well as the hippocampi and cervical ganglia of type 1 diabetic mice $[8,62]$, suggesting that insulin deprivation negatively regulates noradrenergic tone. Thus, in addition to generalized neuroprotective attributes, insulin/IGF signaling can directly influence synaptic transmission.

\section{Hsp interdependency with insulin and IGF-I signaling}

The stability, solubility and signaling capabilities of several insulin/IGF-I signaling components depend upon Hsp interactions. For example, maturation of the insulin receptor hinges upon Hsp90-mediated trafficking of the pro-receptor through the endoplasmic reticulum [63]. Mutations within RTK domains are common in patients with severe insulin resistance and Hsp90 exports these dysfunctional receptors to the cytoplasm for proteolytic degradation [9,11]. Additional Hsp90 client proteins include IGF-IR, IRS-2,

phosphoinositide-dependent kinase-1, Akt, Raf-1, MEK-1 (MAPK/ERK kinase 1), ERK-1, PKC and GSK-3 $\beta$ [10,64-71]. Hsp90 also binds raptor to enhance mTOR signaling [72]. 
Although pro-apoptotic GSK-3 $\beta$ can be reduced with Hsp90 inhibition, the collateral damage to other signaling components negates any potential therapeutic value and inhibition actually worsens disease pathology [73].

Alterations in insulin/IGF-I signaling also impact upon Hsp expression and function. For instance, Hsp27 is stored in an $800 \mathrm{kDa}$ multimeric state. Akt and PKC assist in the dynamic cycling of phosphorylation states required to reduce these Hsp27 multimers into functional oligomeric/monomeric species [74]. Furthermore, progressive HSF1 phosphorylation by ERK-1 (Ser307) and then GSK-3 $\beta$ (Ser303) was recently thought to suppress the HSR [75]. Although this phosphorylation is now known to be GSK-3 $\beta$ independent under normal conditions, assorted physiological stresses may induce allosteric changes in HSF1 that enable GSK-3 $\beta$-mediated phosphorylation at Ser303 or nearby residues [76]. These data suggest that an interdependent relationship exists between insulin/ IGF-I signaling and functional Hsp expression.

\section{Pathological insulin and IGF-I signal disruption}

\section{Ligand and receptor depletion}

In patients with type 1 diabetes, pancreatic $\beta$ cells and/or circulating insulin are selectively targeted through immune-mediated elimination, destroying the majority of systemic insulin supply. Interestingly, antibodies against Hsp90 have been found in some type 1 diabetic patients, suggesting that systemic deficiencies in Hsp90 may be associated with the development of diabetes [77]. In bacteria, the Hsp70 ortholog DnaK can bind three different regions of human proinsulin (but not mature insulin) implicated in autoimmune elimination [78]. Thus, the depletion of Hsp70 may unmask and increase epitope exposure/recognition by $\mathrm{T}$ cells, accelerating the pathophysiological progression of type 1 diabetes [78].

$\mathrm{AD}$ has been proposed as a 'type 3 diabetes' [46]. In $\mathrm{AD}$ patient brains, postmortem analyses of IR and IGF-IR mRNA levels have demonstrated an eight- to tenfold decrease in hippocampal and hypothalamic neurons and a 40\% IR/IGF-IR mRNA drop in the prefrontal cortex, areas implicated in AD pathology [46]. Substantial reductions in the mRNA of insulin (hippocampus: fourfold; hypothalamus: twofold), IGF-I (hypothalamus and prefrontal cortex: five-to sixfold), and IGF-II (hippocampus and hypothalamus: threefold) were also observed [46]. The timeline of these reductions directly corresponds to the Braak stages of AD pathological progression [79]. Observed increases of IGF-I levels within the serum and CSF of AD patients are believed to be attributed to glial secretion in response to neurodegeneration [80].

In PD, IR mRNA declines within the substantia nigra pars compacta, a brain area implicated in PD pathology, without affecting localized insulin levels [49]. Although IR/IGF-IR levels are not altered in people with HD, 33-50\% of these patients develop abnormal glucose tolerance, suggestive of altered insulin efficacy [49].

\section{Pathological disruption of signaling components and Hsp intervention}

Several mutations contributing to insulin resistance in type 2 diabetes (e.g. Akt-2 mutations) have been identified [11]. Grote and coworkers demonstrated that IRS-2 phosphorylation at Ser731 is elevated in the DRG of both type 1 and type 2 diabetic mice, whereas phosphorylated Akt decreased [81]. Ser731 phosphorylation is believed to be inhibitory and is thought to destabilize IRS-2:receptor associations, leading to IRS-2 degradation and diminished signaling (e.g. in adipose and muscle tissue) [81]. Type 2 diabetic mice also displayed impaired insulin-induced neurite outgrowth, suggesting a potential breakdown in insulin-associated repair mechanisms [4,81]. Similarly, chronic insulin treatment of isolated DRG neurons (mimicking hyperinsulinemia) reduced insulin-stimulated Akt activation [82]. 
Collectively, these data suggest that peripheral neurons, as with metabolic tissues, can develop insulin resistance that compromises neurotrophic support and may contribute to the development of DPN.

We have shown that induction of Hsp70 via the C-terminal Hsp90 inhibitor KU-32 reversed several clinical indices of DPN without increasing overall insulin levels [34]. Although the mechanism of action of KU-32 remains under investigation, it seems prudent to speculate that its elicited effects may involve the reconstitution of impaired insulin/IGF signaling components in an Hsp70-dependent manner [34]. As such, the co-administration of other traditional diabetic treatments (e.g. insulin and insulin analogs, secretagogs, metformin or thiazolidinediones) with HSR inducers may synergistically improve DPN. However, this synergy may develop similar risks to those associated with mismanaged insulin therapy if the compound is a potent and broad inducer of molecular chaperones. Additional limitations for HSR induction may include enhanced tumorigenesis because IGF-I signaling and Hsps are significantly upregulated in several forms of cancer [83]. However, such obstacles may be circumvented by readjusting primary treatment regimens and adjuvant therapies (diabetic and other chemotherapeutics) or developing Hsp90 inhibitors that promote a less robust HSR, which is an attribute of KU-32 [34].

In AD models, treating cultured hippocampal neurons with $\mathrm{A} \beta$-derived diffusible ligand (ADDL) decreases IR expression by activating CaMKII (calcium calmodulin-dependent kinase II)- and CK2 (casein kinase II)-mediated receptor internalization [84]. Inversely, activation of IR signaling decreases ADDL binding and associated oxidative stress, an effect further amplified by the insulin-sensitizer rosiglitazone [84]. Therefore, negating ADDL neurotoxicity probably requires functional insulin signaling and does not rely upon competitive binding [84]. Clearance of $A \beta$ from the brain to the periphery is also facilitated through IGF-I administration, which induces the translocation of albumin and transthyretin carrier proteins [85].

$\mathrm{A} \beta_{1-42}$ treatment of rat primary cortical neurons activates GSK-3 $\beta$, enhancing tau phosphorylation, cytochrome $c$ release, caspase-3 activation and HSF1 depletion [86]. Selective inhibition of GSK-3 $\beta$ reverses these effects in a dose-dependent manner [86]. A $\beta$ also activates c-Jun N-terminal kinase (JNK), which phosphorylates and inhibits IRS-1/2 [87]. Abnormally high JNK levels are also implicated in the development of insulin resistance [87]. Furthermore, the pathological progression of tauopathy-prone pR5 mice is significantly enhanced in diabetic (STZ-induced) pR5 mice versus control mice [88]. Tau hyperphosphorylation in wild-type diabetic mice is comparatively less than diabetic pR5 mice, suggesting that insulin depletion enhances, but does not initiate, tauopathy [89]. In addition, ERK- $1 / 2$ activation suppresses $\beta$-secretase (BACE) expression in neuroblastoma cells, thereby reducing $A \beta_{1-42}$ production [90]. Phosphorylated ERK-1/2 levels increase with $\mathrm{AD}$ pathology, suggesting its role as a natural defensive mechanism against accumulating $A \beta_{1-42}$ [90]. These studies collectively suggest that restoring insulin/IGF-I signaling can reduce the expression of $A \beta_{1-42}$ and hyperphosphorylation of tau that are implicated in AD pathology.

Hsps not only enhance insulin/IGF-I signaling component expression and function but also directly interact with $A \beta$ and tau to negate their pathological effects. For example, Hsp90, Hsp70 and Hsp40 bind to and prevent early phases of $A \beta_{1-42}$ self-assembly in vitro [25]. Cterminal Hsp90 inhibitors also prevent $\mathrm{A} \beta_{25-35}$-induced neurotoxicity in SH-SY5Y neuroblastoma cells $[32,33]$. BAG-1- and BAG-2-mediated tau degradation is also enhanced through Hsp70 induction [42]. Although Hsp70 reduces JNK activation in sympathetic neurons, its effects upon JNK activation within AD brains remain to be determined [91]. As such, endogenous upregulation of Hsps should prevent $A \beta$ and tau-induced neurotoxicity. 
However, proteomic analyses of $\mathrm{AD}$ patient hippocampi have revealed only modest increases in Hsp70 [92]. In patients with mild cognitive impairment (MCI), Hsp70 and Hsp27 are elevated within the inferior parietal lobule (IPL), an area implicated in memory retrieval [93]. This same region also demonstrates a 70\% increase in Hsp70 carbonyl levels, suggesting that oxidative damage of Hsp70 may antagonize endogenous upregulation efforts [94]. MEK1 oxidation was also observed, further indicating insulin signal disruption [94]. Histochemical analyses of brains from $\mathrm{rTg} 4510 \mathrm{AD}$ mice indicates a substantial induction of Hsp70 and Hsp27 (but not Hsp90) with age and pathological progression [95]. A more intense and larger Hsp70 band also accrues along the same timeline, suggesting covalent modification (perhaps ubiquitination) of Hsp70 [95]. The induction of Hsp27 (also observed in the hippocampus and caudate putamen) suggests the employment of contingent clearance mechanisms to mitigate compromised or exhausted Hsp70-mediated avenues [42,95]. Therefore, pharmacological HSR induction may expedite the reinforcement needed to negate $\mathrm{A} \beta$-induced insulin/IGF-I signal transduction and facilitate aberrant protein clearance.

\section{Concluding remarks}

In summary, insulin and IGF-I signaling are essential to maintain neuronal viability and synaptic transmission. Induction of the pro-survival HSR enhances numerous insulin and IGF-I signaling components and synergistically mitigates numerous disease-specific aberrant proteins that interfere with this signaling. The development of non-cytotoxic Cterminal Hsp90 inhibitors enables potentially more nuanced exploitation of the HSR. Such inhibitors have already proved to be effective towards treating DPN [34]. Insulin and IGF-I deficiencies provide an interconnecting link between the pathologies of DPN, AD, PD, and HD. The pharmacological development of new HSR inducers may provide a multifaceted therapeutic approach for treating several insulin-associated neurodegenerative disorders.

\section{Acknowledgments}

This work was supported by grants from the Juvenile Diabetes Research Foundation (R.T.D.) and the National institutes of Health (NS058847, R.T.D.) (CA120458, B.S.J.B.).

\section{References}

1. Obrosova IG. Diabetic painful and insensate neuropathy: pathogenesis and potential treatments. Neurotherapeutics. 2009; 6:638-647. [PubMed: 19789069]

2. Vincent AM, et al. Diabetic neuropathy: cellular mechanisms as therapeutic targets. Nat. Rev. Neurol. 2011; 7:573-583. [PubMed: 21912405]

3. Pasquier F, et al. Diabetes mellitus and dementia. Diabetes Metab. 2006; 32:403-414. [PubMed: 17110895]

4. Guo G, et al. Local insulin and the rapid regrowth of diabetic epidermal axons. Neurobiol. Dis. 2011; 43:414-421. [PubMed: 21530660]

5. Belfiore A, et al. Insulin receptor isoforms and insulin receptor/insulin-like growth factor receptor hybrids in physiology and disease. Endocr. Rev. 2009; 30:586-623. [PubMed: 19752219]

6. Xiang Y, et al. Insulin-like growth factor-1 regulates neurite outgrowth and neuronal migration from organotypic cultured dorsal root ganglion. Int. J. Neurosci. 2011; 121:101-106. [PubMed: 21110707]

7. Sullivan KA, et al. Insulin-like growth factors in the peripheral nervous system. Endocrinology. 2008; 149:5963-5971. [PubMed: 18719018]

8. Bitar MS, Pilcher CW. Diabetes attenuates the response of the lumbospinal noradrenergic system to idazoxan. Pharmacol. Biochem. Behav. 2000; 67:247-255. [PubMed: 11124388]

9. Imamura $\mathrm{T}$, et al. Involvement of heat shock protein 90 in the degradation of mutant insulin receptors by the proteasome. J. Biol. Chem. 1998; 273:11183-11188. [PubMed: 9556607] 
10. Martins AS, et al. A pivotal role for heat shock protein 90 in Ewing sarcoma resistance to antiinsulin-like growth factor 1 receptor treatment: in vitro and in vivo study. Cancer Res. 2008; 68:6260-6270. [PubMed: 18676850]

11. O'Rahilly S. Human genetics illuminates the paths to metabolic disease. Nature. 2009; 462:307314. [PubMed: 19924209]

12. Chaudhury S, et al. Hsp90 as a target for drug development. ChemMedChem. 2006; 1:1331-1340. [PubMed: 17066389]

13. Chiosis G, et al. Emerging Hsp90 inhibitors: from discovery to clinic. Anticancer Agents Med. Chem. 2006; 6:1-8. [PubMed: 16475922]

14. Luo W, et al. Heat shock protein 90 in neurodegenerative diseases. Mol. Neurodegener. 2010; 5:24. [PubMed: 20525284]

15. Peterson LB, Blagg BS. To fold or not to fold: modulation and consequences of Hsp90 inhibition. Future Med. Chem. 2009; 1:267-283. [PubMed: 20161407]

16. Fujimoto M, Nakai A. The heat shock factor family and adaptation to proteotoxic stress. FEBS J. 2010; 277:4112-4125. [PubMed: 20945528]

17. Whitesell L, Lindquist SL. HSP90 and the chaperoning of cancer. Nat. Rev. Cancer. 2005; 5:761772. [PubMed: 16175177]

18. Hadden MK, et al. Geldanamycin, radicicol, and chimeric inhibitors of the Hsp90 N-terminal ATP binding site. Curr. Top. Med. Chem. 2006; 6:1173-1182. [PubMed: 16842154]

19. Anckar J, Sistonen L. Regulation of HSF1 function in the heat stress response: implications in aging and disease. Annu. Rev. Biochem. 2011; 80:1089-1115. [PubMed: 21417720]

20. Whitesell L, Lindquist S. Inhibiting the transcription factor HSF1 as an anticancer strategy. Expert Opin. Ther. Targets. 2009; 13:469-478. [PubMed: 19335068]

21. Blagg BS, Kerr TD. Hsp90 inhibitors: small molecules that transform the Hsp90 protein folding machinery into a catalyst for protein degradation. Med. Res. Rev. 2006; 26:310-338. [PubMed: 16385472]

22. Hayden MR, et al. Type 2 diabetes mellitus as a conformational disease. JOP. 2005; 6:287-302. [PubMed: 16006679]

23. Amolins MW, Blagg BS. Natural product inhibitors of Hsp90: potential leads for drug discovery. Mini Rev. Med. Chem. 2009; 9:140-152. [PubMed: 19200020]

24. Matts RL, et al. A systematic protocol for the characterization of Hsp90 modulators. Bioorg. Med. Chem. 2011; 19:684-692. [PubMed: 21129982]

25. Evans CG, et al. Heat shock proteins 70 and 90 inhibit early stages of amyloid beta-(1-42) aggregation in vitro. J. Biol. Chem. 2006; 281:33182-33191. [PubMed: 16973602]

26. Sittler A, et al. Geldanamycin activates a heat shock response and inhibits huntingtin aggregation in a cell culture model of Huntington's disease. Hum. Mol. Genet. 2001; 10:1307-1315. [PubMed: 11406612]

27. Luo W, et al. Roles of heat-shock protein 90 in maintaining and facilitating the neurodegenerative phenotype in tauopathies. Proc. Natl. Acad. Sci. U.S.A. 2007; 104:9511-9516. [PubMed: 17517623]

28. Dickey CA, et al. The high-affinity HSP90-CHIP complex recognizes and selectively degrades phosphorylated tau client proteins. J. Clin. Invest. 2007; 117:648-658. [PubMed: 17304350]

29. Waza M, et al. 17-AAG, an Hsp90 inhibitor, ameliorates polyglutamine-mediated motor neuron degeneration. Nat. Med. 2005; 11:1088-1095. [PubMed: 16155577]

30. Marcu MG, et al. The heat shock protein 90 antagonist novobiocin interacts with a previously unrecognized ATP-binding domain in the carboxyl terminus of the chaperone. J. Biol. Chem. 2000; 275:37181-37186. [PubMed: 10945979]

31. Marcu MG, et al. Novobiocin and related coumarins and depletion of heat shock protein 90dependent signaling proteins. J. Natl. Cancer Inst. 2000; 92:242-248. [PubMed: 10655441]

32. Ansar S, et al. A non-toxic Hsp90 inhibitor protects neurons from Abeta-induced toxicity. Bioorg. Med. Chem. Lett. 2007; 17:1984-1990. [PubMed: 17276679]

33. Lu Y, et al. Neuroprotective activity and evaluation of Hsp90 inhibitors in an immortalized neuronal cell line. Bioorg. Med. Chem. 2009; 17:1709-1715. [PubMed: 19138859] 
34. Urban MJ, et al. Inhibiting heat-shock protein 90 reverses sensory hypoalgesia in diabetic mice. ASN Neuro. 2010; 2:189-199.

35. Neef DW, et al. Modulation of heat shock transcription factor 1 as a therapeutic target for small molecule intervention in neurodegenerative disease. PLoS Biol. 2010; 8:e1000291. [PubMed: 20098725]

36. Liu AY, et al. Neuroprotective drug riluzole amplifies the heat shock factor 1 (HSF1)- and glutamate transporter 1 (GLT1)-dependent cytoprotective mechanisms for neuronal survival. J. Biol. Chem. 2011; 286:2785-2794. [PubMed: 21098017]

37. Brodsky JL, Chiosis G. Hsp70 molecular chaperones: emerging roles in human disease and identification of small molecule modulators. Curr. Top. Med. Chem. 2006; 6:1215-1225. [PubMed: 16842158]

38. Page TJ, et al. Genome-wide analysis of human HSF1 signaling reveals a transcriptional program linked to cellular adaptation and survival. Mol. Biosyst. 2006; 2:627-639. [PubMed: 17216044]

39. Hayashida N, et al. Heat shock factor 1 ameliorates proteotoxicity in cooperation with the transcription factor NFAT. EMBO J. 2010; 29:3459-3469. [PubMed: 20834230]

40. Zhao X, et al. Biological roles of neural J proteins. Cell. Mol. Life Sci. 2008; 65:2385-2396. [PubMed: 18438606]

41. Pratt WB, et al. Proposal for a role of the Hsp90/Hsp70-based chaperone machinery in making triage decisions when proteins undergo oxidative and toxic damage. Exp. Biol. Med. 2010; 235:278-289.

42. Carrettiero DC, et al. The cochaperone BAG2 sweeps paired helical filament- insoluble tau from the microtubule. J. Neurosci. 2009; 29:2151-2161. [PubMed: 19228967]

43. Evans CG, et al. Heat shock protein 70 (hsp70) as an emerging drug target. J. Med. Chem. 2010; 53:4585-4602. [PubMed: 20334364]

44. Havrankova J, et al. Concentrations of insulin and insulin receptors in the brain are independent of peripheral insulin levels.Studies of obese and streptozotocin-treated rodents. J. Clin. Invest. 1979; 64:636-642. [PubMed: 156737]

45. Wallum BJ, et al. Cerebrospinal fluid insulin levels increase during intravenous insulin infusions in man. J. Clin. Endocrinol. Metab. 1987; 64:190-194. [PubMed: 3536982]

46. Steen E, et al. Impaired insulin and insulin-like growth factor expression and signaling mechanisms in Alzheimer's disease - is this type 3 diabetes? J. Alzheimers Dis. 2005; 7:63-80. [PubMed: 15750215]

47. Torres-Aleman I. Toward a comprehensive neurobiology of IGF-I. Dev. Neurobiol. 2010; 70:384396. [PubMed: 20186710]

48. Chen DY, et al. A critical role for IGF-II in memory consolidation and enhancement. Nature. 2011; 469:491-497. [PubMed: 21270887]

49. Craft S, Watson GS. Insulin and neurodegenerative disease: shared and specific mechanisms. Lancet Neurol. 2004; 3:169-178. [PubMed: 14980532]

50. Freude $S$, et al. The role of IGF-1 receptor and insulin receptor signaling for the pathogenesis of Alzheimer's disease: from model organisms to human disease. Curr. Alzheimer Res. 2009; 6:213223. [PubMed: 19519303]

51. Benyoucef S, et al. Characterization of insulin/IGF hybrid receptors: contributions of the insulin receptor L2 and Fn1 domains and the alternatively spliced exon 11 sequence to ligand binding and receptor activation. Biochem. J. 2007; 403:603-613. [PubMed: 17291192]

52. Denley A, et al. Structural determinants for high-affinity binding of insulin-like growth factor II to insulin receptor (IR)-A, the exon 11 minus isoform of the IR. Mol. Endocrinol. 2004; 18:25022512. [PubMed: 15205474]

53. Feng Z, Levine AJ. The regulation of energy metabolism and the IGF-1/mTOR pathways by the p53 protein. Trends Cell Biol. 2010; 20:427-434. [PubMed: 20399660]

54. Evans GJ, et al. Protein kinase B/Akt is a novel cysteine string protein kinase that regulates exocytosis release kinetics and quantal size. J. Biol. Chem. 2006; 281:1564-1572. [PubMed: 16243840] 
55. Linseman DA, et al. Glycogen synthase kinase-3beta phosphorylates Bax and promotes its mitochondrial localization during neuronal apoptosis. J. Neurosci. 2004; 24:9993-10002. [PubMed: 15525785]

56. Li JQ, et al. Regulation of increased glutamatergic input to spinal dorsal horn neurons by mGluR5 in diabetic neuropathic pain. J. Neurochem. 2010; 112:162-172. [PubMed: 19840219]

57. Daulhac L, et al. Phosphorylation of spinal $N$-methyl-D-aspartate receptor NR1 subunits by extracellular signal-regulated kinase in dorsal horn neurons and microglia contributes to diabetesinduced painful neuropathy. Eur. J. Pain. 2011; 15:169, e161-169, e112. [PubMed: 20594879]

58. Wang XL, et al. Downregulation of GABAB receptors in the spinal cord dorsal horn in diabetic neuropathy. Neurosci. Lett. 2011; 490:112-115. [PubMed: 21184807]

59. Yashiro K, Philpot BD. Regulation of NMDA receptor subunit expression and its implications for LTD, LTP, and metaplasticity. Neuropharmacology. 2008; 55:1081-1094. [PubMed: 18755202]

60. Skeberdis VA, et al. Insulin promotes rapid delivery of $N$-methyl-D-aspartate receptors to the cell surface by exocytosis. Proc. Natl. Acad. Sci. U.S.A. 2001; 98:3561-3566. [PubMed: 11248117]

61. Texido L, et al. Amyloid beta peptide oligomers directly activate NMDA receptors. Cell Calcium. 2011; 49:184-190. [PubMed: 21349580]

62. Robertson SD, et al. Insulin reveals Akt signaling as a novel regulator of norepinephrine transporter trafficking and norepinephrine homeostasis. J. Neurosci. 2010; 30:11305-11316. [PubMed: 20739551]

63. Ramos RR, et al. Calreticulin and Hsp90 stabilize the human insulin receptor and promote its mobility in the endoplasmic reticulum. Proc. Natl. Acad. Sci. U.S.A. 2007; 104:10470-10475. [PubMed: 17563366]

64. Basso AD, et al. Akt forms an intracellular complex with heat shock protein 90 (Hsp90) and Cdc37 and is destabilized by inhibitors of Hsp90 function. J. Biol. Chem. 2002; 277:39858-39866. [PubMed: 12176997]

65. Fujita N, et al. Involvement of Hsp90 in signaling and stability of 3-phosphoinositide-dependent kinase-1. J. Biol. Chem. 2002; 277:10346-10353. [PubMed: 11779851]

66. Gould CM, et al. The chaperones Hsp90 and Cdc37 mediate the maturation and stabilization of protein kinase $\mathrm{C}$ through a conserved PXXP motif in the C-terminal tail. J. Biol. Chem. 2009; 284:4921-4935. [PubMed: 19091746]

67. Lochhead PA, et al. A chaperone-dependent GSK3beta transitional intermediate mediates activation-loop autophosphorylation. Mol. Cell. 2006; 24:627-633. [PubMed: 17188038]

68. Schulte TW, et al. Destabilization of Raf-1 by geldanamycin leads to disruption of the Raf-1MEK-mitogen-activated protein kinase signalling pathway. Mol. Cell Biol. 1996; 16:5839-5845. [PubMed: 8816498]

69. Fukushima T, et al. HSP90 interacting with IRS-2 is involved in cAMP-dependent potentiation of IGF-I signals in FRTL-5 cells. Mol. Cell. Endocrinol. 2011; 344:81-89. [PubMed: 21742014]

70. Stancato LF, et al. The hsp90-binding antibiotic geldanamycin decreases Raf levels and epidermal growth factor signaling without disrupting formation of signaling complexes or reducing the specific enzymatic activity of Raf kinase. J. Biol. Chem. 1997; 272:4013-4020. [PubMed: 9020108]

71. Taherian A, et al. A comparison of Hsp90alpha and Hsp90beta interactions with cochaperones and substrates. Biochem. Cell Biol. 2008; 86:37-45. [PubMed: 18364744]

72. Ohji G, et al. Suppression of the mTOR-raptor signaling pathway by the inhibitor of heat shock protein 90 geldanamycin. J. Biochem. 2006; 139:129-135. [PubMed: 16428328]

73. Yoshikawa N, et al. Distinct regulation of insulin receptor substrate-1 and -2 by $90-k D a$ heat-shock protein in adrenal chromaffin cells. Neurochem. Int. 2010; 56:42-50. [PubMed: 19737590]

74. Abisambra JF, et al. Phosphorylation dynamics regulate Hsp27-mediated rescue of neuronal plasticity deficits in tau transgenic mice. J. Neurosci. 2010; 30:15374-15382. [PubMed: 21084594]

75. Frame S, Cohen P. GSK3 takes centre stage more than 20 years after its discovery. Biochem. J. 2001; 359:1-16. [PubMed: 11563964]

76. Batista-Nascimento L, et al. Deciphering human heat shock transcription factor 1 regulation via post-translational modification in yeast. PLoS ONE. 2011; 6:e15976. [PubMed: 21253609] 
77. Qin HY, et al. Type 1 diabetes alters anti-hsp90 autoantibody isotype. J. Autoimmun. 2003; 20:237-245. [PubMed: 12753809]

78. Burkart V, et al. High affinity binding of hydrophobic and autoantigenic regions of proinsulin to the $70 \mathrm{kDa}$ chaperone DnaK. BMC Biochem. 2010; 11:44. [PubMed: 21059249]

79. Rivera EJ, et al. Insulin and insulin-like growth factor expression and function deteriorate with progression of Alzheimer's disease: link to brain reductions in acetylcholine. J. Alzheimers Dis. 2005; 8:247-268. [PubMed: 16340083]

80. Salehi Z, et al. Insulin like growth factor-1 and insulin like growth factor binding proteins in the cerebrospinal fluid and serum from patients with Alzheimer's disease. Biofactors. 2008; 33:99_ 106. [PubMed: 19346585]

81. Grote $\mathrm{CW}$, et al. Insulin receptor substrate 2 expression and involvement in neuronal insulin resistance in diabetic neuropathy. Exp. Diabetes Res. 2011; 2011:212571. [PubMed: 21754917]

82. Kim B, et al. Hyperinsulinemia induces insulin resistance in dorsal root ganglion neurons. Endocrinology. 2011; 152:3638-3647. [PubMed: 21810948]

83. Breinig M, et al. Heat shock protein 90-sheltered overexpression of insulin-like growth factor 1 receptor contributes to malignancy of thymic epithelial tumors. Clin. Cancer Res. 2011; 17:22372249. [PubMed: 21372220]

84. De Felice FG, et al. Protection of synapses against Alzheimer's-linked toxins: insulin signaling prevents the pathogenic binding of Abeta oligomers. Proc. Natl. Acad. Sci. U.S.A. 2009; 106:1971-1976. [PubMed: 19188609]

85. Carro E, et al. Serum insulin-like growth factor I regulates brain amyloid-beta levels. Nat. Med. 2002; 8:1390-1397. [PubMed: 12415260]

86. Koh SH, et al. Amyloid-beta-induced neurotoxicity is reduced by inhibition of glycogen synthase kinase-3. Brain Res. 2008; 1188:254-262. [PubMed: 18031715]

87. Ma QL, et al. Beta-amyloid oligomers induce phosphorylation of tau and inactivation of insulin receptor substrate via c-Jun N-terminal kinase signaling: suppression by omega-3 fatty acids and curcumin. J. Neurosci. 2009; 29:9078-9089. [PubMed: 19605645]

88. Ke YD, et al. Experimental diabetes mellitus exacerbates tau pathology in a transgenic mouse model of Alzheimer's disease. PLoS ONE. 2009; 4:e7917. [PubMed: 19936237]

89. Moroz N, et al. Limited Alzheimer-type neurodegeneration in experimental obesity and type 2 diabetes mellitus. J. Alzheimers Dis. 2008; 15:29-44. [PubMed: 18780965]

90. Tamagno E, et al. JNK and ERK1/2 pathways have a dual opposite effect on the expression of BACE1. Neurobiol. Aging. 2009; 30:1563-1573. [PubMed: 18255190]

91. Bienemann AS, et al. Hsp70 suppresses apoptosis in sympathetic neurones by preventing the activation of c-Jun. J. Neurochem. 2008; 104:271-278. [PubMed: 17971127]

92. Sultana R, et al. Proteomics analysis of the Alzheimer's disease hippocampal proteome. J. Alzheimers Dis. 2007; 11:153-164. [PubMed: 17522440]

93. Di Domenico F, et al. Protein levels of heat shock proteins 27, 32, 60, 70, 90 and thioredoxin-1 in amnestic mild cognitive impairment: an investigation on the role of cellular stress response in the progression of Alzheimer disease. Brain Res. 2010; 1333:72-81. [PubMed: 20362559]

94. Sultana R, et al. Redox proteomic analysis of carbonylated brain proteins in mild cognitive impairment and early Alzheimer's disease. Antioxid. Redox Signal. 2010; 12:327-336. [PubMed: 19686046]

95. Dickey C, et al. Aging analysis reveals slowed tau turnover and enhanced stress response in a mouse model of tauopathy. Am. J. Pathol. 2009; 174:228-238. [PubMed: 19074615] 


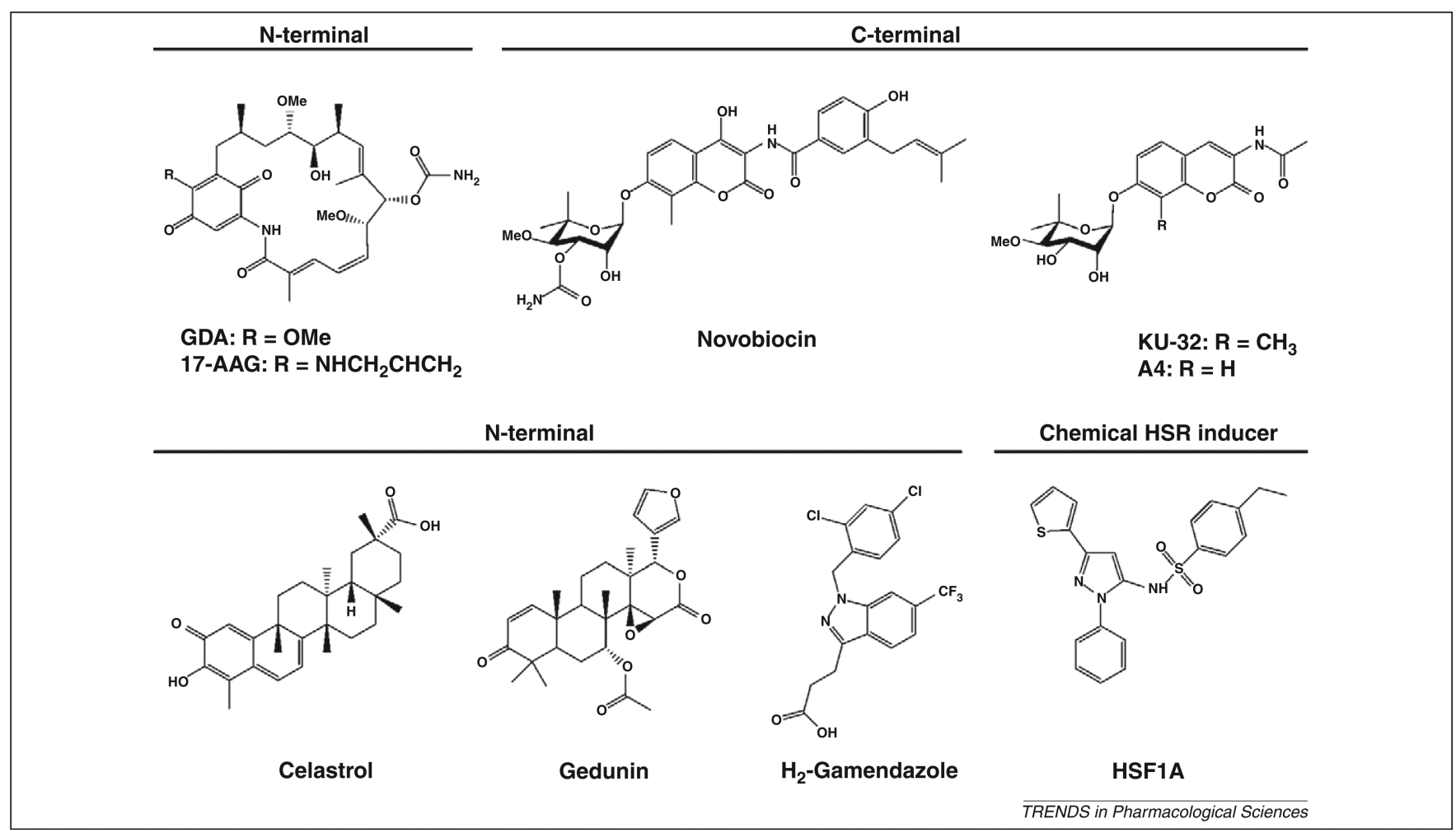

Figure 1.

Structures of N-terminal Hsp90 inhibitors (GDA, 17-AAG, Celastrol, Gedunin, and $\mathrm{H}_{2}$ Gamendazole), C-terminal Hsp90 inhibitors (KU-32 and A4), and chemical HSR inducer HSF1A. 


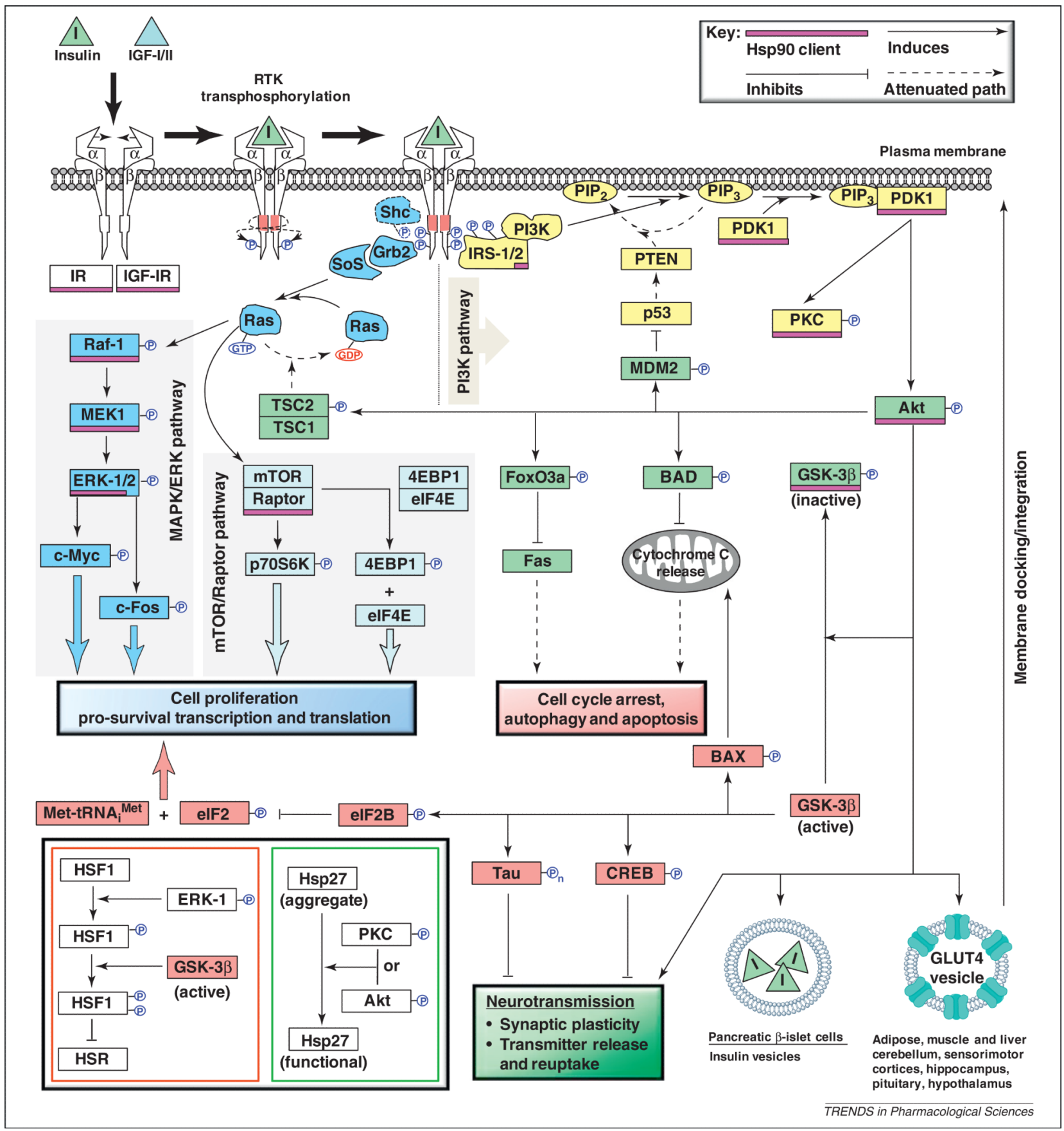

Figure 2.

Insulin, IGF-I and IGF-II binding to IR and IGF-IR. Ligand binding triggers receptor autophosphorylation and RTK activation, permitting IRS-1, IRS-2 or Shc phosphorylation. Mitogenic signaling pursues via Grb2 activation of the GEF (guanine nucleotide exchange factor) SoS, enabling Ras/Rheb activation. Activated Ras/Rheb initiates the MAPK/ERK pathway through Raf-1 phosphorylation and enhances mTOR/raptor signaling, collectively increasing cell growth, proliferation, protein expression and viability. Induction of the PI3K pathway enhances metabolic insulin effects and mitigates pro-apoptotic and anti-mitogenic signaling. Hsp90 client proteins are indicated with a pink bar and serve crucial roles in regulating insulin/IGF signaling. Potential modulation effects of insulin/IGF signaling 
components upon Hsp expression and function are shown in the black box. Abbreviations: $\mathrm{BAD}, \mathrm{Bcl}$-2-associated death promoter; BAX, Bcl-2-associated X; 4EBP1, eIF4E binding protein-1; CREB, cAMP-response element binding; eIF2, eukaryotic initiation factor 2; eIF4E, eukaryotic translation initiation factor-4E; ERK-1/2, extracellular-signal-regulated kinase-1/2; FoxO3a, forkhead box 'other' 3a; GLUT4, glucose transporter 4; Grb2, growth factor receptor-bound protein 2; GSK-3 $\beta$, glycogen synthase kinase-3 $\beta$; HSF1, heat shock factor-1; Hsp, heat shock protein; HSR, heat shock response; IGF-IR, insulin-like growth factor-I receptor; IR, insulin receptor; IRS-1/2, insulin receptor substrate-1/2; MAPK, mitogen-activated protein kinase; MEK1, MAPK/ERK kinase 1; Met-tRNA $\mathrm{A}_{\mathrm{i}}^{\text {Met }}$, initiator methionyl-tRNA; mTOR, mammalian target of rapamycin; raptor, regulatory-associated protein of mTOR; MDM2, murine double minute-2; PDK1, phosphoinositide-dependent kinase-1; PI3K, phosphatidylinositol 3-kinase; $\mathrm{PIP}_{2}$, phosphatidylinositol 4,5-bisphosphate; $\mathrm{PIP}_{3}$, phosphatidylinositol 3,4,5-triphosphate; PKC, protein kinase C; PTEN, phosphatase and tensin homolog; RTK, receptor tyrosine kinase; Shc, Src homology 2 domaincontaining; SoS, son of sevenless; TSC, tuberous sclerosis complex. 
Table 1

IR and IGF-IR distribution patterns within the nervous system

\begin{tabular}{l|l|l|l|l}
\hline Receptor & Central nervous system & Refs & Peripheral nervous system & Refs \\
\hline IR & Cerebral cortex & {$[49]$} & Sensory neurons & {$[7]$} \\
\hline & Hippocampus & & Sympathetic neurons & \\
\hline & Amygdala & & & \\
\hline & Striatum & & & \\
\hline & Substantia nigra & & & \\
\hline & Hypothalamus & & & \\
\hline & Thalamus & & & \\
\hline & Cerebellum & & & \\
\hline & Pons & & & \\
\hline & Medulla & & & \\
\hline & Cervical spinal cord & & & \\
\hline & Hippocampus (adult) & {$[7,50]$} & Motor neurons & \\
\hline & Amygdala (adult) & & Sensory neurons & \\
\hline & Parahippocampal gyrus (adult) & & Schwann cells & \\
\hline
\end{tabular}

${ }^{a}$ Ubiquitously expressed throughout most tissues, but most concentrated in the regions listed. 
Table 2

Insulin, IGF-I and IGF-II IC $50(\mathrm{nM})$ values $^{a}$

\begin{tabular}{l|l|l}
\hline Isoform & Ligand $\left(\right.$ IC $\left._{\mathbf{5 0}}, \mathbf{n M}\right)$ & Ref. \\
\hline IR-A $^{b}$ & Insulin $(0.2)<$ IGF-II $(2.2)<$ IGF-I (9.0) & {$[51]$} \\
\hline IR-B & Insulin $(0.5)<$ IGF-II $(10.0)<$ IGF-I (90.0) & {$[51]$} \\
\hline IGF-IR & IGF-I $(0.8)<$ IGF-II (4.4) < Insulin $(>100)$ & {$[52]$} \\
\hline
\end{tabular}

${ }^{a}$ Adapted from Belfiore et al. [5].

${ }^{b}$ Receptor lacks exon 11, enhancing ligand binding affinity. 